
\title{
R Reserach S Suare \\ Efficacy of a Thermally Triggered Injectable Hydrogel CS/Col /PLA/GP and BMSCs for Cartilage Tissue Engineering
}

\section{Mingjing Li}

Wuhan fourth hospital;Puai hospital,Tongji medical college,Huazhong university of science and technology

Fan Li ( $\sim$ orthopaedic2020@163.com )

Wuhan No 4 Hospital: Tongji Medical College Affiliated Wuhan Puai Hospital https://orcid.org/00000001-7640-1132

\section{Research}

Keywords: Thermosensitive hydrogel, Chitosan, Type II collagen, Polylactic acid, Cartilage tissue engineering

Posted Date: October 28th, 2020

DOI: https://doi.org/10.21203/rs.3.rs-96928/v1

License: (c) (1) This work is licensed under a Creative Commons Attribution 4.0 International License. Read Full License 


\section{Abstract}

\section{Background}

Articular cartilage has limited self-repair ability. Tissue engineering is considered to be one of the most promising therapeutic approaches. Chitosan (CS) based hydrogels are the most widely used scaffolds which still need improvement. The purpose of this study was to investigate the efficacy of a thermally triggered injectable chitosan / type II collagen / polylactic acid / sodium $\beta$-glycerophosphate (CS/Col/PLA/GP) hydrogel and bone marrow mesenchymal stem cells (BMSCs) for the treatment of cartilage defects in rabbit knee joints.

Material/Methods

The CS-based hydrogels consisting of CS, Col II, PLA and GP were fabricated by chemical cross-linking method. The gel forming time and elastic modulus of these hydrogels were measured. We tested the viability, proliferation and differentiation of rabbit BMSCs cultured in the hydrogels by fluorescence staining, CCK-8 and PCR method. The hydrogels combined with or without BMSCs were injected into cartilage defects in rabbit knee joints and the materials were collected at 8 weeks after surgery. The repair effect of cartilage defects was evaluated based on gross observation, $\mathrm{HE}$, safranin $\mathrm{O}$ and immunohistochemical staining.

Results

The CS/Col/PLA/GP hydrogel was liquid at room temperature and gelled after $7.5 \pm 0.41 \mathrm{~min}$ at $37^{\circ} \mathrm{C}$. $\mathrm{CS} / \mathrm{Col} / \mathrm{PLA} / \mathrm{GP}$ hydrogel had a modulus of $8.90 \pm 0.12 \mathrm{kPa}$ while CS/GP and CS/Col/GP hydrogels had the modulus of $4.07 \pm 0.24 \mathrm{kPa}$ and $4.93 \pm 0.09 \mathrm{kPa}$. The results of Live/Dead cell viability assay reveal that most of BMSCs remained alive in the hydrogels. CCK-8 assay shows that the number of cells in CS/Col /PLA/GP hydrogel was significantly higher in comparison to the other groups on days 2 and 3 of cell culture $(p<0.05)$. Aggrecan mRNA expression in the CS/Col /PLA/GP gel was the highest $(p<0.05)$. Sox9 mRNA expression in the CS/Col /GP group was the highest, in which CS/Col /PLA/GP hydrogel was higher than the CS/GP hydrogel(p<0.05). Furthermore, CS/Col/PLA/GP and CS/Col /GP hydrogels showed higher COL2A1 mRNA expression in comparison to CS/GP constructs $(p<0.05)$. In vivo studies showed that approximately $90 \%$ of the cartilage defects of rabbits treated by the hydrogel and BMSCs were repaired with hyaline-like tissue without obvious inflammation response. $\mathrm{HE}$, safranin $\mathrm{O}$, and immunohistochemical staining showed that the hyaline like cartilage was formed in cartilage defects, and the collagen content in the new generated cartilage was similar to the normal cartilage. The neocartilage was thinner than the surrounding normal cartilage, but it exhibited integration with adjacent healthy tissue. The abundant well-defined chondrocytes were aligned in several apparent chondrocyte clusters in the new generated cartilage.

Conclusions 
The thermo-sensitive injectable CS/Col/PLA/GP composite hydrogel has better ability to promote survive, proliferation and chondrogenic differentiation of seeded BMSCs as compared against CS/Col/GP and CS/GP hydrogels. Combined with BMSCs to repair cartilage defects of rabbit knee joints, they can effectively reduce the cartilage defect area, and the new generated cartilage is comparable to normal cartilage structure. In addition, abundant availability and simple fabrication process also make CS/Col/PLA/GP composite hydrogel a suitable candidate scaffold in cartilage tissue engineering.

\section{Background}

Articular cartilage defects appear with frequency in orthopaedic clinical practice as result of trauma, infections or degeneration of joints[1]. Even a minor defect may fail to heal, thus degenerating to osteoarthritis, which leads to knee joint dysfunction, significant pain, and even disability[2, 3]. Articular cartilage defects have been treated with microfracture, autologous chondrocyte implantation and bone marrow stimulation [4]. However, there are problems such as inaccurate clinical efficacy, secondary damage to the donor area, and limited availability[4] . In recent years, cartilage tissue engineering has become a significant alternative to conventional treatment[5].

Tissue engineering to promote cartilage regeneration must take on three elements: scaffolds, cells, and growth agents[6]. Designing appropriate material for tissue engineering is a critical step in tissue engineering. Recently, hydrogels have been considered one of the most promising materials because they could offer high water content, implantation without open surgery, and in situ scaffold formation [7]. In particular, in situ cross-linkable hydrogels have been widely used as therapeutic implants for cartilage tissue engineering[8]. They can be injected into the target sites using injectable devices and can encapsulate cells or drugs during hydrogel formation.

Natural polymer derived chitosan(CS) hydrogel has recently been paid much attention due to its advantages such as biocompatibility, biodegradability and antibacterial activity[9] . CS binds to sodium $\beta$ glycerophosphate (GP) and generates thermosensitive gel complex[10] . The hydrogel displayed a desirable gelling behavior that is useful for intra-articular injection (liquid-like at room temperature and gel-like at $37^{\circ} \mathrm{C}$ ). They are able to fill abnormally shaped defects and support intra-operative cell seeding through minimally invasive methods, which greatly facilitate clinical applications.

Although CS hydrogels have numerous advantages, their mechanical and electrical properties are not good enough to satisfy cartilage tissue engineering. It is sometimes not possible to prepare a biomaterial with desired properties using only one polymer. Therefore, composite materials are created where a supportive material can be added to comply with the necessary mechanical properties [11]. It was shown that freeze-dried CS/gelatin scaffolds crosslinked with genipin support bone regeneration in vivo in mice, inducing extracellular matrix (ECM) production with minimal inflammatory reactions [12]. The addition of TEMPO-oxidized cellulose nanofiber induced faster gelation and increased porosity with improved biocompatibility in vitro and in vivo in comparison to CS[13]. It was known that synthetic thermoplastic 
polymers such as polycaprolactone (PCL), polylactic acid (PLA), have mechanical properties well suited for use as scaffold structures for cartilage tissue engineering as they are relatively stiff and strong[14]. It was also demonstrated that CS /PLA hybrid scaffold improved stiffness in comparison to CS hydrogel alone. The low mechanical properties of hydrogels can be overcome with the use of PLA which improve the mechanical stability of the hydrogel[15]. However, they undergo rapid cell-mediated contraction and generate fibrous cartilage rather than hyaline cartilage. It is still a challenge to mimic native cartilage more accurately and to improve their mechanical and biological properties.

It is well-known that type II collagen (Col II) is the main component of articular cartilage. It was found that the hydrogel prepared by collagen is rich in bioactive sites beneficial to the growth of cells and tissues, and has good biocompatibility [16]. It has been reported that Col II hydrogels resulted in the more prominent chondrogenic differentiation of MSC, compared to alginate or type I collagen [17]. Zhang and his colleagues designed a biocompatible bone repair material prepared from crosslinked porous constructs of collagen and hydroxyapatite [18]. The results in their research demonstrated the novel materials presented high biocompatibility and effectively induced osteanagenesis ability. CS could interact with collagen via electrostatic interactions between abundant amino groups and sulfo groups [19], and freeze-dried Col II /CS hybrid scaffold possesses improved stiffness in comparison to single component scaffolds, with a good porous structure resembling cartilage[20].

Based on previous research, we have designed a novel thermosensitive, injectable hydrogel scaffold which includes CS, Col II, PLA and GP. BMSCs, combined with CS/Col /PLA/GP hydrogel, were injected into the articular cartilage defects in a rabbit model. We evaluated physical and biological properties of the new fabricated hydrogel and determined the efficacy of cartilage defects treatment with this hydrogel and BMSCs.

\section{Results}

\subsection{Physical properties of the CS-based hydrogels}

All CS-based hydrogels were liquid at room temperature and irreversibly gelled following incubation at $37^{\circ} \mathrm{C}$ in $10 \mathrm{~min}$ but the time durations were different (Figure 1). All formulations were light yellow and clear. CS/GP and CS/Col /GP solution became gel in $8.87 \pm 0.48 \mathrm{~min}$ and $8.0 \pm 0.41 \mathrm{~min}$, while CS/Col /PLA/GP solution became gel in $7.5 \pm 0.41 \mathrm{~min}$ (Figure 1B). The difference between three groups was not statistically significant $(p>0.05)$. Results of compression test on the hydrogels were shown in Figures $1 \mathrm{C}$. The compressive modules of the hydrogels increased significantly after the PLA and Col were supplemented, compared with CS gel alone. It revealed that CS/Col /PLA/GP hydrogel was 2.19 times stiffer than CS/GP hydrogel $(p<0.05)$. CS/Col /PLA/GP hydrogel had a modulus of $8.90 \pm 0.12 \mathrm{kPa}$ while $\mathrm{CS} / \mathrm{GP}$ and CS/Col/GP hydrogels had the modulus of $4.07 \pm 0.24 \mathrm{kPa}$ and $4.93 \pm 0.09 \mathrm{kPa}$.

\subsection{Survival, proliferation and differentiation results of BMSCs in the CS-based hydrogels}


BMSCs were cultured in the three CS-based hydrogels for 3 days and then stained with the Live/Dead staining kit. The results are shown in Figure 2. It can be seen that there is almost no red fluorescence in the field of 400x and 600x, and the entire field of view is bright green. It revealed that most of BMSCs remained alive in the hydrogels.

Figure 3 shows the results of CCK-8 assay. As it is shown, three CS-based hydrogels improve and accelerate BMSCs proliferation on day 1, 2, and 3 after culturing in the gels. There was no significant difference in the number of cells between the groups on the first day. After 2 or 3 days, cell proliferations in hydrogels were significantly higher in comparison to the control group $(p<0.05)$. Among these three hydrogels, the CS/Col /PLA/GP group has the strongest cell proliferation ability.

The qRT-PCR technique was used to detect the changes in the expression level of BMSCs chondrogenic differentiation-related mRNA. The results are shown in Figure 2F: The expression of chondrogenic differentiation-related mRNA showed a certain difference. Aggrecan mRNA expression in the CS/Col /PLA/GP gel was the highest with statistical significance $(p<0.05)$. Sox 9 mRNA expression in the CS/Col /GP group was the highest, in which CS/Col /PLA/GP hydrogel was higher than the CS/GP hydrogel $(\mathrm{p}<0.05)$. Furthermore, CS/Col /PLA/GP and CS/Col /GP hydrogels showed higher COL2A1 mRNA expression in comparison to CS/GP constructs $(p<0.05)$. There was no significant difference between the CS/Col /PLA/GP group and the CS/Col /GP group. The results indicate CS/Col /PLA/GP hydrogel has the best biocompatibility, which not only favors the survival and proliferation of BMSCs, but also helps them differentiate into chondrocytes.

\subsection{Macroscopic observations of the articular cartilage}

Figure 5B shows that the cartilage defects in CS/Col /PLA/GP hydrogel+BMSCs group generally exhibited cartilage-like formation at 8 weeks after surgery. Approximately $90 \%$ of the defect was repaired with hyaline-like tissue and the joint surface was relatively smooth. The margin was integrated with adjacent healthy tissue (Figure 5B4). No signs of cartilage erosion, or synovial proliferation were observed in the operated knees. The repair effects in the hydrogel+BMSCs group were remarkably better than those in the control (Figure 5B2) and hydrogel groups (Figure 5B3). The surgical defects with hydrogel implantation at 8 weeks had irregular surfaces with fibrosis and were incompletely healed. The defects in the control

group were still concave, irregular, or empty in the middle and the boundary was clear (Figure 5B2).

According to the ICRS scores from macroscopic observations, the average scores in the hydrogel+BMSCs group $(10.33 \pm 1.51)$ were higher than those in the control group $(2.83 \pm 0.75)$ and hydrogel group (5.17 \pm 0.75$)$ (Figure 5). The scoring showed a significant difference among those three groups $(P<0.05)$, which means that the application of CS/Col/PLA/GP hydrogel to fill cartilage defects favor cartilage repair, but hydrogel combined with BMSCs has the best repair effect.

\subsection{Histological and immunohistochemical evaluations of the articular cartilage}


Figure 6 shows the results of $\mathrm{HE}$, safranin $\mathrm{O}$, immunohistochemical staining of Col II of the new generated cartilage. In hydrogel+BMSCs group, the cartilage defects were repaired by hyaline-like cartilage at 8 weeks (Figure 6 D1-D6). The neocartilage was thinner than the surrounding normal cartilage, but it exhibited integration with adjacent healthy tissue. The abundant well-defined chondrocytes were aligned in several apparent chondrocyte clusters in the new generated cartilage which had integrated with subchondral bone. The Col II content in the new generated cartilage was similar to the normal cartilage. No obvious inflammatory response and hydrogel residue were observed. In hrdrogel group, more fibrocartilage and less integration were discovered in cartilage defects (Figure 6 C1-C6). In control group, the defects were filled with severe fibrosis, and no cartilage was discovered (Figure 6 B1B6).

\section{Discussion}

With its limited self-repair potential, human articular cartilage undergoes frequent irreversible damages. The repair of cartilage defects remains a significant challenge in orthopedic medicine. Cartilage tissue engineering based on materials science and cell biology was considered to be one of the most promising therapeutic approaches for cartilage defects [4].

Environmentally sensitive hydrogels are one of the important components of cartilage tissue engineering scaffolds [5]. They show a phase transition form a solution to a gel state via various physical and chemical stimuli. According to the cross-linking strategies, they can be divided into photo-sensitive, thermo-sensitive, $\mathrm{pH}$-sensitive hydrogels and so on [21]. Studies have been conducted to prepare $\mathrm{pH}-$ sensitive hydrogels by reaction between dibenzaldehyde terminated PEG and chitosan[22] . Some researchers prepared the photosensitive hydrogel by introducing the chlorophyll photosensitive group into the PNIPAm hydrogel [23]. The CS/Col /PLA/GP hydrogel in this article has thermo-sensitive property. It is liquid at room temperature and forms a gel at $37^{\circ} \mathrm{C}$ after 7-8 minutes. Thermo-sensitive characteristics and suitable gelation time make it suitable for intra-articular injection and in-situ gelation in the defect area. Compared with other types of smart hydrogels, this hydrogel does not depend on $\mathrm{pH}$ or light with special wavelengths, and can undergo a liquid-gel phase transition at physiological temperature. At the same time, the thermo-sensitive gel is formed by physical cross-linking, avoiding the disadvantages of using chemical cross-linking agents. The results in vivo suggested that CS/Col /PLA/GP solution was injected into the cartilage defects of the rabbit, and a gel formed after about 8 minutes. No shedding occurred, and the cartilage defect area was completely filled with hydrogel. In clinical treatment, the hydrogel solution can be injected into cartilage defects of different sizes and shapes through arthroscopy or other minimally invasive methods, and the gel can be formed in situ by itself to exert a repairing effect, which greatly facilitates clinical practical applications.

CS/GP hydrogel is a weak gel with a very low compression modulus. This study shows that the elastic modulus of the hybrid scaffold significantly increased when compared to CS/GP gel alone (4kPa), after the PLA and Col II were supplemented. Wu Jingjing and others designed and synthesized chitosan/silk fibroin/nano-hydroxyapatite composite gel by adding nano-hydroxyapatite to significantly improve the 
mechanical properties, and the elastic modulus can reach more than $40 \mathrm{kPa}$ [24]. However, BMSCs still showed desired proliferation and differentiation into chondrocytes in the CS/Col II /PLA/GP gel, which may be related to the lower mechanical properties of the gel. As we known, tissue elasticity can range from less than $1 \mathrm{KPa}$ (brain, lung), $1 \mathrm{MPa}$ (cartilage), up to 2-4 GPa (bone)[25]. Some scaffolds with poor mechanical properties still display excellent prospects in applications $[26,27]$. Previous studies have reported when gel rigidity increased, although overall cell survival decreased, more osteogenic differentiation of MSCs was observed[28]. Some researchers have reported that BMSCs showed osteogenic differentiation in the bioglass-alginate scaffold with strong mechanical properties [29]. Some studies have demonstrated that BMSCs cultured in calcium phosphate scaffolds embedded in CS hydrogels increase cartilage matrix components synthesis and chondrogenic diffrentiation, in comparison to calcium phosphate scaffolds alone[30]. Engler and other scholars found that mechanical properties of the biomaterials affect cell behaviour[31]. Consistent with our results, growing evidence has demonstrated that MSCs differentiate into neural, muscle, cartilage lineages after being cultured on the soft substrates, while MSCs differentiate into bone lineages on the stiffer substrates [31].

In addition to the mechanical properties, the composition and structure of scaffolds affect the synthesis and quality of the new generated cartilage. Specifically, recent researches have demonstrated that the differentiation capability of stem cells is enhanced in a three dimensional (3D) culture system both in vivo and in vitro[32] . Compared with other scaffolds, hydrogel has a spatial structure more similar to extracellular matrix. In addition to water, the extracellular matrix is mainly composed of collagen, proteoglycans and other protein macromolecules [1]. In this study, type II collagen contained in the $\mathrm{CS} / \mathrm{Col} / \mathrm{PLA} / \mathrm{GP}$ composite gel is the main component of articular cartilage. CS is a linear carbohydrate biopolymer with a structural similarity to glycosaminoglycans of the extracellular matrix (ECM). Some researchers have reported that the collagen component provides contact with a native ECM protein that regulates the adhesion, proliferation, and differentiation of MSC[33]. Type II collagen hydrogels have been shown to promote chondrogenic differentiation of embedded MSCs[34] .It was reported that chondrocytes embedded in collagen gel maintained the cartilage phenotype in long-term cultures [35]. The results in this study showed that BMSCs can proliferate and differentiate into chondrocyte in the CS/Col /PLA/GP composite hydrogel. 8 weeks after surgery, it was observed that all the hydrogels were completely degraded in the cartilage defect area without significant inflammatory response. Hyaline-like tissues were generated, which were almost flush with the surrounding normal cartilage tissue. No obvious hydrogel residue was observed in histological staining. Some researchers combined chondrocytes with type $₫$ collagen and implanted them into the cartilage defects of rabbits. Consistent with our results, it can be seen that the tissue engineered cartilage is similar in height and texture to the surrounding normal cartilage after 12 weeks [5]. Some studies have wrapped chondrocytes with hyaluronic acid composite hydrogel, and found that it can maintain the phenotype and high activity of cartilage cells, and increase the expression of cartilage matrix [36].In another study, in vivo experiments for the repair of cartilage defects in rabbits using gelatin-polylactic acid multilayer gel and BMSCs have shown the formation of hyaline cartilage by the fourth week post-implantation [37]. The results of this study showed that the new generated cartilage staining in hydrogel+BMSCs group is close to the surrounding tissue, and 
chondrocytes can be seen in it. Similar to other researches, the thickness of new generated cartilage in this study is insufficient, and the degree of integration with normal cartilage needs to be further improved. How to adopt more innovative engineering approaches to generate more complex tissue constructs with better mechanical and biological properties, and extend the duration of observation in animal experiments are all aspects that need to be improved upon the future.

\section{Conclusions}

In summary, the thermo-sensitive injectable CS/Col /PLA/GP composite hydrogel has better ability to promote survive, proliferation and chondrogenic differentiation of seeded BMSCs as compared against CS/Col/GP and CS/GP hydrogels. Combined with BMSCs to repair cartilage defects of rabbit knee joints, they can effectively reduce the cartilage defect area, and the new generated cartilage is comparable to normal cartilage structure. In addition, abundant availability and simple fabrication process also make $\mathrm{CS} / \mathrm{Col} / \mathrm{PLA} / \mathrm{GP}$ composite hydrogel a suitable candidate scaffold in cartilage tissue engineering.

\section{Materials And Methods}

\subsection{Materials}

New Zealand white rabbits (4-5 months old, weighing 2.5-3.0 kg, provided by the Animal Experiment Center of Tongji Medical College); Chitosan (molecular weight 21,000Da, deacetylation degree $>95 \%$, Jinan Haidebei Marine Biological Engineering, China), sodiumß-glycerophosphate(Aladdin, USA), Acetic acid(chemical reagents of analytical grade, Sigma, USA), Type II Collagen (Shanghai Xiangduo Biological, China), Polylactic Acid (Sigma, USA) , 囚-MEM medium (Gibco, USA), DMEM / F12 medium (Gibco, USA), fetal bovine serum (Gibco, USA), 0.25\% trypsin (containing EDTA) (Gibco, USA), Live/Dead cell staining kit(Sigma, USA), type II Collagenase and trypsin (Sigma, USA), HE and Safranin O staining kit (Gibco, USA), anti-type II collagen antibody (Abcam, USA), goat anti-rabbit horseradish peroxidase IgG (Abcam, USA), SYBR ${ }^{\circledR}$ Green master mix (Applied Biosystems, Foster City, CA); Fluorescence inverted microscope (Olympus, Japan), Universal tensile testing machine『SANS CMT4000, MTS, China『.

\subsection{Fabrication of thermosensitiveCS-based hydrogels}

Firstly, $2000 \mathrm{mg}$ of CS powder was dissolved in $100 \mathrm{~mL}$ acetic acid $(0.1 \mathrm{~mol} / \mathrm{L})$ and vigorously stir for $1 \mathrm{~h}$ with $300 \mathrm{rpm}$ to prepare a $2 \%$ solution. The solution were then passed through a gauze filter, sterilized by autoclave $\left(121^{\circ} \mathrm{C}, 15 \mathrm{~min}\right)$ and stored at $4{ }^{\circ} \mathrm{C} ; 25 \mathrm{mg}$ Col II was dissolved in $1.25 \mathrm{~mL}$ acetic acid $(0.1 \mathrm{~mol} /$ L) to prepare a $2 \%$ solution; $20 \mathrm{mg}$ PLA was dissolved in $1 \mathrm{~mL}$ chloroform solution to prepare a $2 \%$ solution; $5600 \mathrm{mg}$ GP powder was dissolved in $10 \mathrm{~mL}$ distilled water to prepare a $56 \%$ solution, which was sterilized by suction filtration. Secondly, the hydrogel was prepared by mixing CS with Col II and PLA at the ratio of 2: 1: 1; the pre-cooled GP was added dropwise to the mixed solution in the ice bath under constant stirring with $300 \mathrm{rpm}$ to reach a final concentration of $6 \%$. The CS/GP, CS/Col /GP hydrogels were prepared following the same steps above and served as controls. 


\subsection{Gelation time determination and mechanical characterization of CS-based hydrogels}

The test tube inverting method was used to measure the gelation time at constant temperature of $37^{\circ} \mathrm{C}$ in a water bath. $1 \mathrm{~mL}$ of each sample $(n=5)$ of hydrogel solution was added into test tubes at room temperature before incubated in water bath. The fluidity of the samples was observed every $30 \mathrm{~s}$ by tilting the tube. The time at which flow stopped was taken as the gelation time and the values were recorded.

Compression tests were carried out by a universal tensile testing machine. At least four specimens were tested for each group. Samples were cut into cubic specimens and compressed by two parallel metal platens. The state of the hydrogel samples was examined with crosshead speed of $2 \mathrm{~mm} / \mathrm{min}$ until reaching $20 \%$ strain. Force and deformation data were collected by the test instrument and were converted to stress and strain values. The elastic modulus was calculated from the slope of the initial linear segment of stress-strain curves.

\subsection{In vitro culture of BMSCs in CS-based hydrogels}

The animal experiment was carried out in accordance with relevant guidelines and regulations, and was approved by the Medical Ethics Committee of the Puai Hospital affiliated to Tongji Medical College, Huazhong University of Science and Technology (number: KY-2020-106-01). Three New Zealand rabbits were sacrificed under general anaesthesia by injection of $30 \mathrm{mg} / \mathrm{kg}$ sodium pentobarbital (1\%) through the auricular veins. The femur and tibia were isolated under aseptic conditions and the muscles on the bone surface were removed. BMSCs were isolated as published previously[38]. BMSCs were cultured in DMEM supplemented with $10 \%$ FBS and $1 \%$ antibiotic-antimycotic solution (penicillin, streptomycin, amphotericin, and gentamycin) under standard cell culture conditions $\left(37^{\circ} \mathrm{C}, 5 \% \mathrm{CO}_{2}, 95 \%\right.$ humidity). The first medium change was performed after $24 \mathrm{~h}$, and then the medium was changed every half a day for half a volume. BMSCs were passaged and expanded. All experiments were performed with passage 3 (P3) BMSCs.

The sterile hydrogels were prepared as previously mentioned in an aseptic environment and mixed with BMSCs suspension of appropriate amount reaching a final cell density of $2 * 10^{5}$ cells $/ \mathrm{mL}$. They were placed in 24-well plates $(0.5 \mathrm{~mL}$ for each well). To induce thermal gelation, hydrogels were placed in an incubator at $37^{\circ} \mathrm{C}$ for 10 mins. After gelation, all the hydrogels were washed three times with cell culture medium every 30 mins. $1 \mathrm{~mL}$ complete medium was added to each well and changed every 2 days.

\subsection{Cell viability and proliferation assessments of BMSCs seeded in hydrogels}

Cell viability seeded in hydrogels was evaluated using Live/Dead cell viability assay. After 3 days of incubation, cell-seeded scaffolds were washed in PBS for three times. Each constructs was immersed in $500 \mathrm{uL}$ of PBS with Calcein AM $(0.05 \% \mathrm{v} / \mathrm{v})$ and ethidium homodimer $1(0.2 \% \mathrm{v} / \mathrm{v})$ and incubated for $2 \mathrm{~h}$ at $37^{\circ} \mathrm{C}$. Samples were imaged using a fluorescence inverted microscope applying an excitation/emission wave length of $350 / 460 \mathrm{~nm}$. 
Cell proliferation was determined using the Cell Counting Kit-8 (CCK-8, Sigma-Aldrich, USA) on days 1, 2, and 3 of cell culture, following manufacturer instructions. 2D culture system was taken as control group. Briefly, cell culture medium of the samples (three replicates) was removed and $350 \mu \mathrm{L}$ fresh culture medium with $35 \mu \mathrm{L}$ CCK-8 reagent was added to each sample. After incubated at $37^{\circ} \mathrm{C}$ for $2 \mathrm{~h}, 100 \mu \mathrm{L}$ medium of each well was transferred to 96 -well plate. The absorbance values were measured using a microplate reader at wave length of $450 \mathrm{~nm}$. The results obtained were expressed as optical density (OD) after blank subtraction.

\subsection{Cartilage-specific gene expression analysis of BMSCs seeded in hydrogels}

On days 21 of cell cultured in chondrogenic medium(DMEM, $10 \mu \mathrm{g} / \mathrm{ml}$ insulin, transferrin, selenium, 0.1 $\mu \mathrm{M}$ dexamethasone, $40 \mu \mathrm{g} / \mathrm{mL}$ proline, $50 \mu \mathrm{g} / \mathrm{mL}$ ascorbate-2-phopshate, $10 \mathrm{ng} / \mathrm{mL}$ TGF- $\beta 3$ ), total RNA was collected from constructs by sequential use of Trizol and RNeasy mini kit plus, according to the manufacturer's instructions. 500 ng total RNA was reverse transcribed into cDNA using SuperScript III first-strand synthesis kit (Thermo Fisher Scientific). Quantitative real-time polymerase chain reaction (qRTPCR) was performed using SYBR ${ }^{\circledR}$ Green master mix on a StepOnePlus Real-Time PCR system (Applied Biosystems). GAPDH was used as the housekeeping gene. Relative genes expression (Sox9, Aggrecan, COL2A1) were assessed using the $\triangle \triangle C T$ method. Primer sequences are as follows: GAPDH (Forward: CAAGGCTGAGAACGGGAAGC; Reverse: AGGGGGCAGAGATGATGACC), Sox9 (Forward: CTGAGCAGCGACGTCATCTC; Reverse: GTTGGGCGGCAGGTACTG), Aggrecan (Forward: GCTACACTGGCGAGCACTGTAACAT; Reverse: GCGCCAGTTCTCAAATTGCATGGG), COL2A1 (Forward: GCTGGTGAAGAAGGCAAGA; Reverse: AGAACACGGACCACAAGGA).

The mechanical and biological properties were invested and compared for all hydrogels in order to select the most suitable hydrogel for in vivo studies.

\subsection{Construction of rabbit cartilage defects models}

24 New Zealand rabbits were randomly divided into four groups ( $N=6$ per group): A: normal group; $\mathrm{B}$ : control group; C: hydrogel group; D: hydrogel + BMSCs group. The hydrogel with best mechanical and biological properties was selected to take the in vivo studies. Normal group without any treatment was taken as comparison. The rabbits in B, C and D groups were anesthetized by injection of $30 \mathrm{mg} / \mathrm{kg}$ sodium pentobarbital ( $1 \%$ ) through the auricular veins. The knee joints were exposed via a medial parapatellar approach. The knees were flexed to expose the femoral articular cartilage, and a circular fullthickness defect in the articular cartilage was made using a 4.5-mm drill to create a 3-mm-deep hole extended into subchondral bone (Figure 5). The cartilage defect in the control group received no treatment; the hydrogel group was filled with the CS /Col/PLA/GP hydrogel; the hydrogel + BMSCs group was filled with CS /Col/PLA/GP hydrogel and BMSCs. The incision was sutured layer by layer after the hydrogel was solidified. All rabbits were given an injection of $80,000 \mathrm{U}$ of penicillin after operation for three days and allowed to move freely in the cage. No pain killers were used after the surgery. The general conditions of the animals are observed and recorded daily. 


\subsection{Evaluation of efficiency of articular cartilage repair}

At 8 weeks after surgery, the animals were sacrificed by injecting overdose chloral hydrate, and then the knee joints were harvested for further research.

(1) Gross morphology: The entire knees of each rabbit were dissected and the distal part of each femur was extirpated. The samples from each group were photographed and examined for evaluation according to the criteria proposed by the International Cartilage Repair Society (ICRS) [39].

(2) Histology and immunohistochemical staining: All specimens were fixed with $4 \%$ paraformaldehyde solution for $72 \mathrm{~h}$. They were embedded in paraffin after dehydrated routinely, and cut into $5 \mu \mathrm{m}$ thick sections. Sections were stained with hematoxylin-eosin (HE), safranin-0, and immunohistochemical staining of type Il collagen. All specimens were observed using optical microscope.

\subsection{Statistical analysis}

All quantitative results were obtained from at least triplicate measurements. SPSS 18.0 statistical software was used for data analysis. The data were expressed as the mean \pm standard deviation. Comparison between samples was carried out by analysis of variance. P value $<0.05$ was considered statistically significant.

\section{Declarations}

\section{Ethics approval and consent to participate}

The animal experiment was approved by the Medical Ethics Committee of the Puai Hospital affiliated to Tongji Medical College, Huazhong University of Science and Technology (number: KY-2020-106-01).

\section{Consent for publication}

Not applicable.

\section{Competing interests}

The authors declare that they have no competing interests.

\section{Funding}

This research was supported by the Nature Science Foundation from the Science and Technology Department of Hubei Province (grant number: 2017CFB385) and Clinical Medical Research Project from the Health and Family Planning Commission of Wuhan Municipality (grant number: WX17Q20).

\section{Authors' contributions}


All authors have made substantial contribution to the conception, design, performance, analysis of the data, and the drafting of the article revision for important intellectual content.

\section{Acknowledgements}

None

Availability of data and material

All data generated or analysed during this study are included in this published article.

\section{References}

1. Hunziker EB, Lippuner K, Keel MJ, Shintani N: An educational review of cartilage repair: precepts \& practice-myths \& misconceptions-progress \& prospects. (1522-9653 (Electronic)).

2. Brittberg $\mathrm{M}$, Gomoll AH, Canseco JA, Far J, Lind M, Hui J: Cartilage repair in the degenerative ageing knee. (1745-3682 (Electronic)).

3. Eitner A, Hofmann GO, Schaible HG: Mechanisms of Osteoarthritic Pain. Studies in Humans and Experimental Models. (1662-5099 (Print)).

4. Richter DL, Schenck RC, Jr., Wascher DC, Treme G: Knee Articular Cartilage Repair and Restoration Techniques: A Review of the Literature. (1941-0921 (Electronic)).

5. Kwon H, Brown WE, Lee CA, Wang D, Paschos N, Hu JC, Athanasiou KA: Surgical and tissue engineering strategies for articular cartilage and meniscus repair. (1759-4804 (Electronic)).

6. Pierce BF, Tronci G Fau - Rössle M, Rössle M Fau - Neffe AT, Neffe At Fau - Jung F, Jung F Fau Lendlein A, Lendlein A: Photocrosslinked co-networks from glycidylmethacrylated gelatin and poly(ethylene glycol) methacrylates. (1616-5195 (Electronic)).

7. Hoffman AS: Hydrogels for biomedical applications. (0169-409X (Print)).

8. Yu L, Ding J: Injectable hydrogels as unique biomedical materials. (0306-0012 (Print)).

9. Shariatinia Z, Jalali AM: Chitosan-based hydrogels: Preparation, properties and applications. (18790003 (Electronic)).

10. Zhou HY, Jiang LJ, Cao PP, Li JB, Chen XG: Glycerophosphate-based chitosan thermosensitive hydrogels and their biomedical applications. (1879-1344 (Electronic)).

11. Haleem AM, Chu CR: Advances in Tissue Engineering Techniques for Articular Cartilage Repair. (1048-6666 (Print)).

12. Saravanan S, Chawla A, Vairamani M, Sastry TP, Subramanian KS, Selvamurugan N: Scaffolds containing chitosan, gelatin and graphene oxide for bone tissue regeneration in vitro and in vivo. (1879-0003 (Electronic)).

13. Nguyen THM, Abueva C, Ho HV, Lee SY, Lee BT: In vitro and in vivo acute response towards injectable thermosensitive chitosan/TEMPO-oxidized cellulose nanofiber hydrogel. (1879-1344 (Electronic)). 
14. Yao Q, Cosme JG, Xu T, Miszuk JM, Picciani PH, Fong H, Sun H: Three dimensional electrospun PCL/PLA blend nanofibrous scaffolds with significantly improved stem cells osteogenic differentiation and cranial bone formation. (1878-5905 (Electronic)).

15. Haaparanta AM, Järvinen E Fau - Cengiz IF, Cengiz If Fau - Ellä V, Ellä V Fau - Kokkonen HT, Kokkonen Ht Fau - Kiviranta I, Kiviranta I Fau - Kellomäki M, Kellomäki M: Preparation and characterization of collagen/PLA, chitosan/PLA, and collagen/chitosan/PLA hybrid scaffolds for cartilage tissue engineering. (1573-4838 (Electronic)).

16. Lee $\mathrm{CH}$, Singla A Fau - Lee Y, Lee Y: Biomedical applications of collagen. (0378-5173 (Print)).

17. Tiruvannamalai Annamalai R, Mertz DR, Daley EL, Stegemann JP: Collagen Type II enhances chondrogenic differentiation in agarose-based modular microtissues. (1477-2566 (Electronic)).

18. Jiang X, Zhong Y, Zheng L, Zhao J: Nano-hydroxyapatite/collagen film as a favorable substrate to maintain the phenotype and promote the growth of chondrocytes cultured in vitro. (1791-244X (Electronic)).

19. Perez-Puyana V, Jiménez-Rosado M, Romero A, Guerrero A: Crosslinking of hybrid scaffolds produced from collagen and chitosan. (1879-0003 (Electronic)).

20. Levingstone TJ, Matsiko A, Dickson GR, O'Brien FJ, Gleeson JP: A biomimetic multi-layered collagenbased scaffold for osteochondral repair. (1878-7568 (Electronic)).

21. Samchenko Y, Ulberg Z Fau - Korotych O, Korotych O: Multipurpose smart hydrogel systems. (18733727 (Electronic)).

22. Zhang Y, Tao L Fau - Li S, Li S Fau - Wei Y, Wei Y: Synthesis of multiresponsive and dynamic chitosan-based hydrogels for controlled release of bioactive molecules. (1526-4602 (Electronic)).

23. Wells LA, Lasowski F Fau - Fitzpatrick SD, Fitzpatrick Sd Fau - Sheardown H, Sheardown H:

Responding to change: thermo- and photo-responsive polymers as unique biomaterials. (0278-940X (Print)).

24. Wu J, Zheng K, Huang X, Liu J, Liu H, Boccaccini AR, Wan Y, Guo X, Shao Z: Thermally triggered injectable chitosan/silk fibroin/bioactive glass nanoparticle hydrogels for in-situ bone formation in rat calvarial bone defects. (1878-7568 (Electronic)).

25. Discher DE, Mooney Dj Fau - Zandstra PW, Zandstra PW: Growth factors, matrices, and forces combine and control stem cells. (1095-9203 (Electronic)).

26. Gillogly SD, Wheeler KS: Autologous Chondrocyte Implantation With Collagen Membrane. (15381951 (Electronic)).

27. Krill M, Early N, Everhart JS, Flanigan DC: Autologous Chondrocyte Implantation (ACl) for Knee Cartilage Defects: A Review of Indications, Technique, and Outcomes. (2329-9185 (Electronic)).

28. Chaudhuri O, Gu L, Klumpers D, Darnell M, Bencherif SA, Weaver JC, Huebsch N, Lee HP, Lippens E, Duda GN, Mooney DJ: Hydrogels with tunable stress relaxation regulate stem cell fate and activity. (1476-1122 (Print)). 
29. Zhu Y, Kong L, Farhadi F, Xia W, Chang J, He Y, Li H: An injectable continuous stratified structurally and functionally biomimetic construct for enhancing osteochondral regeneration. (1878-5905 (Electronic)).

30. Liu T, Li J, Shao Z, Ma K, Zhang Z, Wang B, Zhang Y: Encapsulation of mesenchymal stem cells in chitosan/ $\beta$-glycerophosphate hydrogel for seeding on a novel calcium phosphate cement scaffold. (1873-4030 (Electronic)).

31. Engler AJ, Sen S Fau - Sweeney HL, Sweeney HI Fau - Discher DE, Discher DE: Matrix elasticity directs stem cell lineage specification. (0092-8674 (Print)).

32. Ravi M, Paramesh V Fau - Kaviya SR, Kaviya Sr Fau - Anuradha E, Anuradha E Fau - Solomon FDP, Solomon FD: 3D cell culture systems: advantages and applications. (1097-4652 (Electronic)).

33. Pulkkinen HJ, Tiitu V Fau - Valonen P, Valonen P Fau - Jurvelin JS, Jurvelin Js Fau - Rieppo L, Rieppo L Fau - Töyräs J, Töyräs J Fau - Silvast TS, Silvast Ts Fau - Lammi MJ, Lammi Mj Fau - Kiviranta I, Kiviranta I: Repair of osteochondral defects with recombinant human type II collagen gel and autologous chondrocytes in rabbit. (1522-9653 (Electronic)).

34. Vázquez-Portalatı NN, Kilmer CE, Panitch A, Liu JC: Characterization of Collagen Type I and II Blended Hydrogels for Articular Cartilage Tissue Engineering. (1526-4602 (Electronic)).

35. Lan W, Xu M, Zhang X, Zhao L, Huang DA-O, Wei X, Chen W: Biomimetic polyvinyl alcohol/type II collagen hydrogels for cartilage tissue engineering. (1568-5624 (Electronic)).

36. Wang G, Cao X, Dong H, Zeng L, Yu C, Chen X: A Hyaluronic Acid Based Injectable Hydrogel Formed via Photo-Crosslinking Reaction and Thermal-Induced Diels-Alder Reaction for Cartilage Tissue Engineering. LID - 10.3390/polym10090949 [doi] LID - 949. (2073-4360 (Electronic)).

37. Ruan SQ, Yan L, Deng J, Huang WL, Jiang DM: Preparation of a biphase composite scaffold and its application in tissue engineering for femoral osteochondral defects in rabbits. (1432-5195 (Electronic)).

38. Maridas DE, Rendina-Ruedy E, Le PT, Rosen CJ: Isolation, Culture, and Differentiation of Bone Marrow Stromal Cells and Osteoclast Progenitors from Mice. LID - 10.3791/56750 [doi] LID - 56750. (1940-087X (Electronic)).

39. Mainil-Varlet P, Aigner T Fau - Brittberg M, Brittberg M Fau - Bullough P, Bullough P Fau - Hollander A, Hollander A Fau - Hunziker E, Hunziker E Fau - Kandel R, Kandel R Fau - Nehrer S, Nehrer S Fau Pritzker K, Pritzker K Fau - Roberts S, Roberts S Fau - Stauffer E, Stauffer E: Histological assessment of cartilage repair: a report by the Histology Endpoint Committee of the International Cartilage Repair Society (ICRS). (0021-9355 (Print)).

\section{Figures}


A

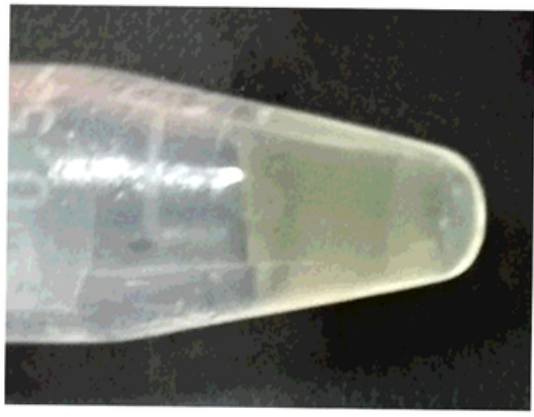

$\mathrm{CS} / \mathrm{GP}$

B

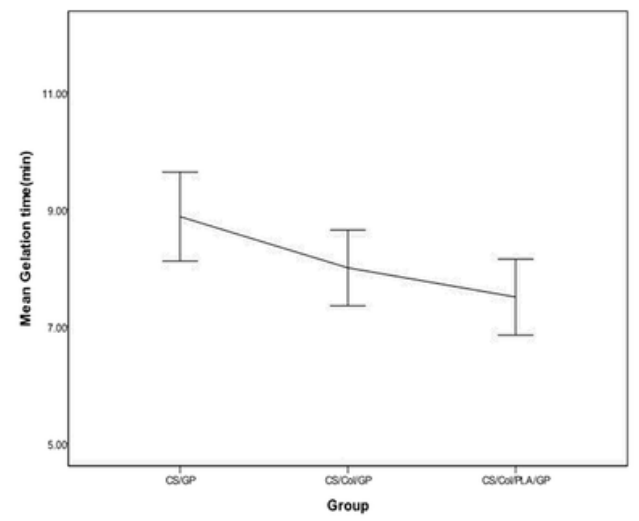

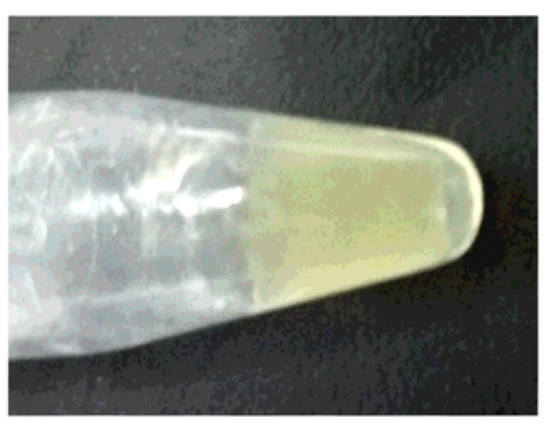

$\mathrm{CS} / \mathrm{Col} / \mathrm{GP}$

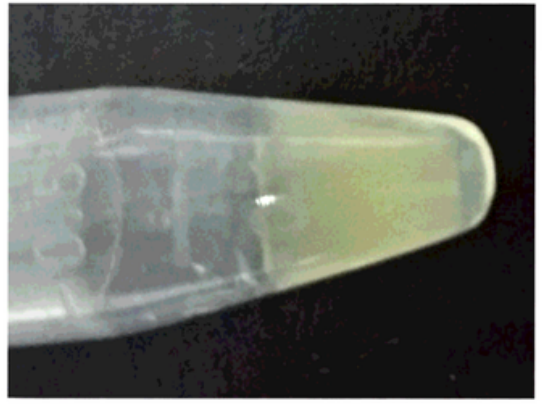

$\mathrm{CS} / \mathrm{Co} 1 / \mathrm{PLA} / \mathrm{GP}$
$\mathrm{C}$

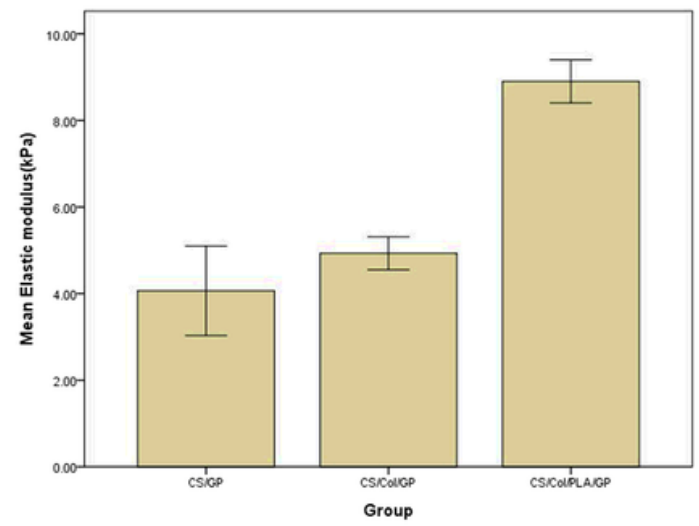

\section{Figure 1}

Physical properties of CS-based hydrogels. (A) Gross images of CS/GP, CS/Col /GP, CS/Col /PLA/GP composite hydrogels after gelation. (B) The gelation time of the three groups of hydrogels was not different ( $p>0.05)$. (C) The elastic modulus of the three groups of hydrogels. The elastic modulus of $\mathrm{CS} / \mathrm{Col} / \mathrm{PLA} / \mathrm{GP}$ hydrogel was higher than that of CS/GP and CS/Col /GP hydrogels $(\mathrm{p}<0.05)$. (CS: chitosan, Col: collagen type II, PLA: polylactic acid, GP: sodium $\beta$-glycerophosphate) 

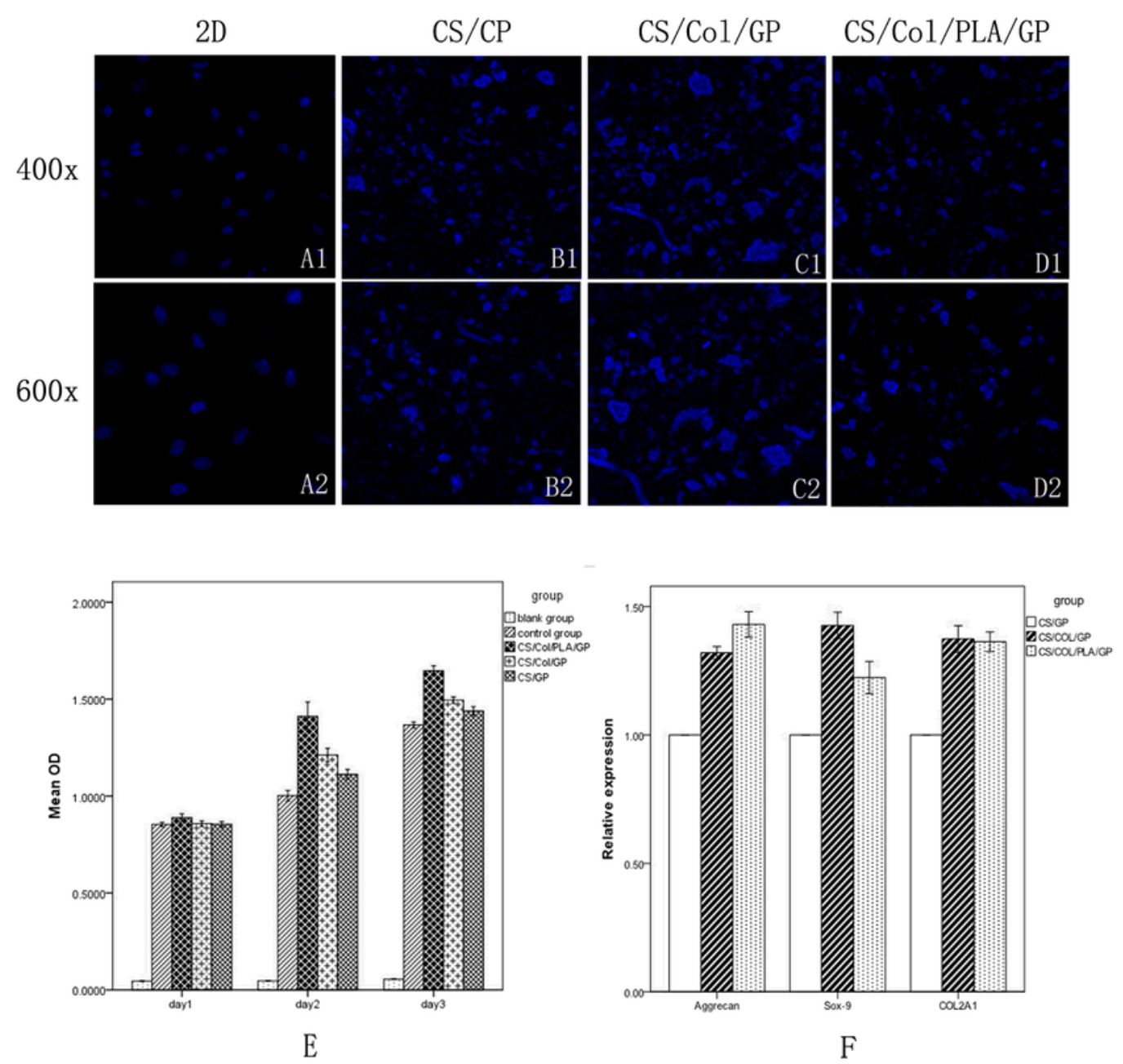

\section{Figure 2}

Biological properties of CS-based hydrogels. A1-D1, A2-D2: The images of the normal group (A1,A2), $\mathrm{CS} / \mathrm{GP}$ hydrogel $(\mathrm{B} 1, \mathrm{~B} 2), \mathrm{CS} / \mathrm{Col} / \mathrm{GP}$ hydrogel $(\mathrm{C} 1, \mathrm{C} 2)$, and CS/Col/PLA/GP hydrogel (D1,D2), magnified 400 and 600 times. E: The comparison of BMSCs proliferation on 2D normal medium (control group) and $\mathrm{CS} / \mathrm{CP}, \mathrm{CS} / \mathrm{Col} / \mathrm{GP}, \mathrm{CS} / \mathrm{Col} / \mathrm{PLA} / \mathrm{GP}$ hydrogels after 1,2 and 3 days $(\mathrm{p}<0.05)$. F: Aggrecan, Sox-9 and COL2A1 gene expressions of BMSCs in each group after three weeks of inducing chondrogenic differentiation $(p<0.05)$. (BMSCs :bone marrow mesenchymal stem cells) 


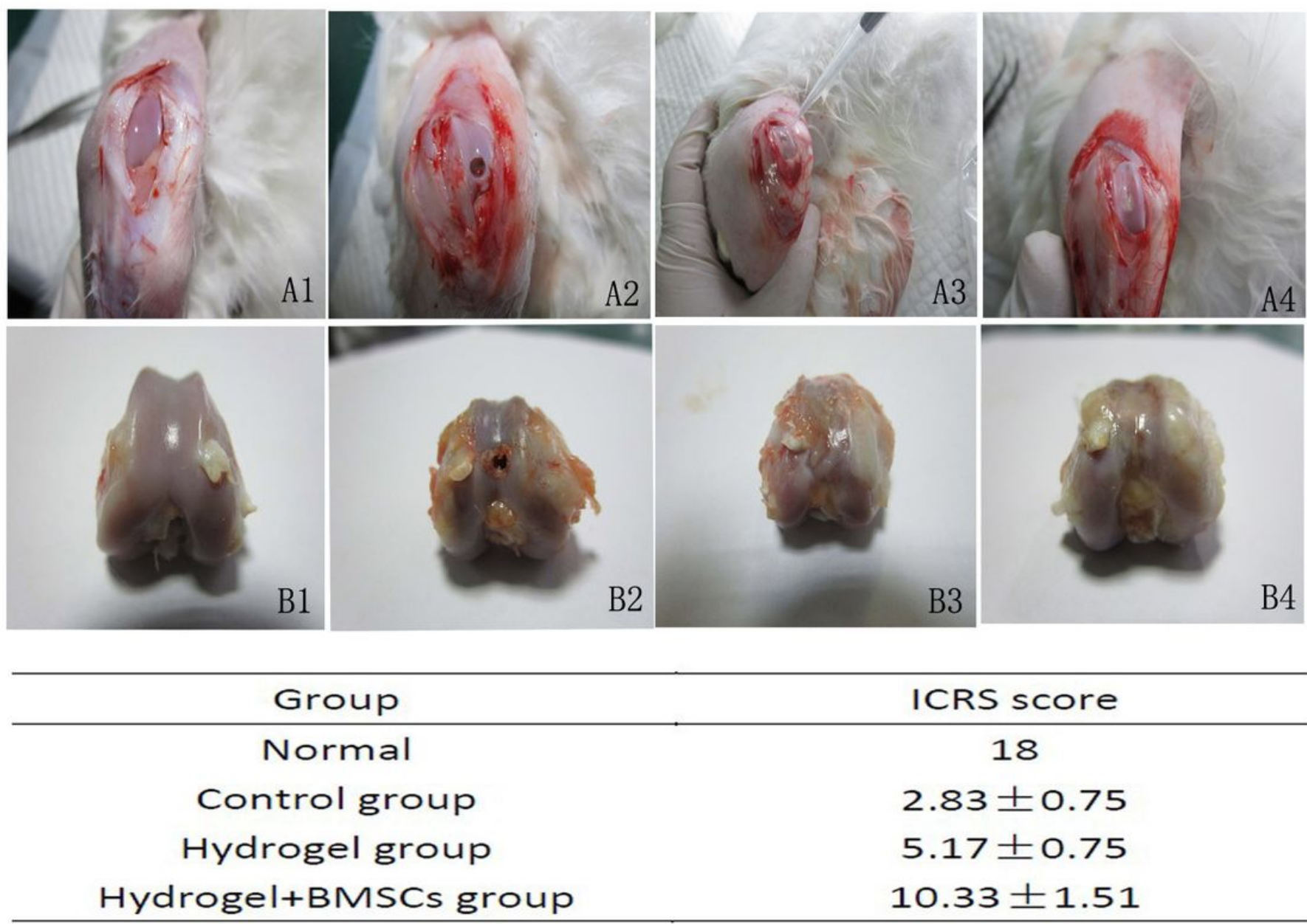

\section{Figure 3}

A1:The knee joints were exposed via a medial parapatellar approach. A2: Cartilage defect with a diameter of $4.5 \mathrm{~mm}$ was created by the electric drill. A3: The cartilage defect was filled with hydrogel by injection. A4: The hydrogel irreversibly gelled at body temperature after 8 minutes. B1-B4: Gross morphology of the joints of the normal group (B1), control group (B2), hydrogel group (B3), and hydrogel+BMSCs group (B4) at 8 weeks after surgery. The bottom table shows the ICRS scores for each group $(p<0.05)$. 


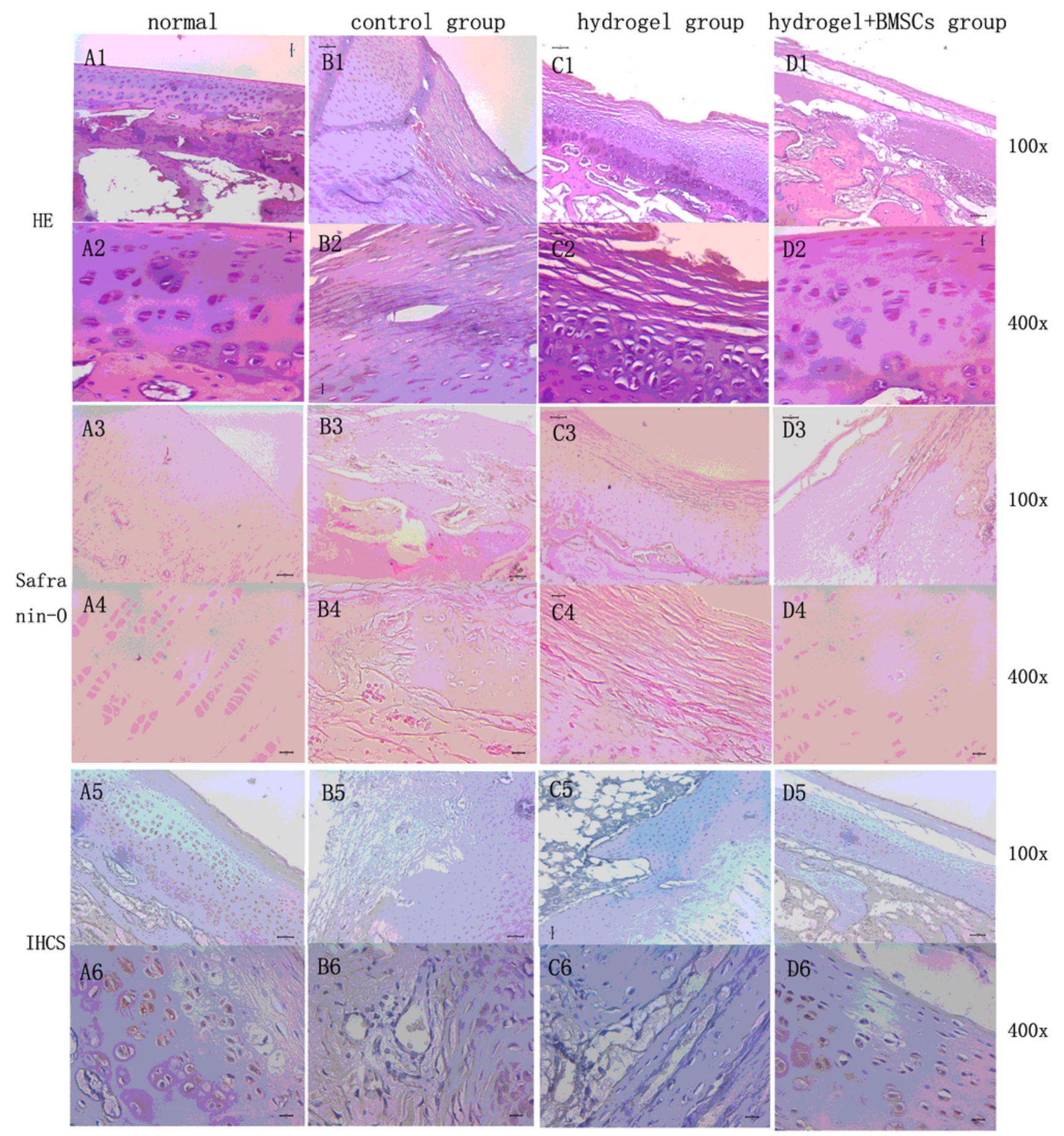

\section{Figure 4}

The images of HE, Safranin $O$ and Collagen type II immunohistochemical staining of cartilage at 8 weeks after surgery. A1-D1, A2-D2: The images of HE staining of the normal group (A1,A2), control group (B1,B2), hydrogel group (C1,C2), and hydrogel+BMSCs group (D1,D2), magnified 100 and 400 times. A3D3, A4-D4: The images of Safranin 0 staining of the normal group (A3,A4), control group (B3,B4), hydrogel group (C3,C4), and hydrogel+BMSCs group (D3,D4), magnified 100 and 400 times. A5-D5, A6- 
D6: The images of Collagen type II immunohistochemical staining of the normal group (A5,A6), control group (B5,B6), hydrogel group (C5,C6), and hydrogel+BMSCs group (D5,D6), magnified 100 and 400 times. Scale bar $=100 \mu \mathrm{m}$. 